\title{
Intermembrane Space of the Mitochondrion
}

National Cancer Institute

\section{Source}

National Cancer Institute. Intermembrane Space of the Mitochondrion. NCI Thesaurus.

Code C32831.

The space between the outer membrane and the inner membrane of the mitochondrion. 